\title{
Research
}

\section{Impact of integrating pharmacists into primary care teams on health systems indicators:}

\author{
a systematic review
}

\begin{abstract}
Background

Evidence suggests that pharmacists integrated into primary care can improve patient outcomes and satisfaction, but their impact on healthcare systems is unclear.

\section{Aim}

To identify the key impacts of pharmacists' integration into primary care on health system indicators, such as healthcare utilisation and costs.
\end{abstract}

\section{Design and setting}

A systematic review of literature.

\section{Method}

Embase, MEDLINE, Scopus, the Health

Management Information Consortium, CINAHL, and the Cochrane Central Register of Controlled Trials databases were examined, along with reference lists of relevant studies. Randomised controlled trials (RCTs) and observational studies published up until June 2018, which considered health system outcomes of the integration of pharmacists into primary care, were included. The Cochrane risk of bias quality assessment tool was used to assess risk of bias for RCTs, the National Institute of Health National Heart, Lung, and Blood Institute quality assessment tool was used for observational studies. Data were extracted from published reports and findings synthesised.

\section{Results}

Searches identified 3058 studies, of which 28 met the inclusion criteria. Most included studies were of fair quality. Pharmacists in primary care resulted in reduced use of GP appointments and reduced emergency department (ED) attendance, but increased overall primary care use. There was no impact on hospitalisations, but some evidence of savings in overall health system and medication costs.

\section{Conclusion}

Integrating pharmacists into primary care may reduce GP workload and ED attendance. However, further higher quality studies are needed, including research to clarify the costeffectiveness of the intervention and the longterm impact on health system outcomes.

\section{Keywords}

family practice; general practice; pharmacists; primary health care; workload.

\section{INTRODUCTION}

Increasingly unmanageable workloads in primary care ${ }^{1}$ makes the recruitment and retention of GPs challenging. ${ }^{2}$ Despite government promises, ${ }^{3}$ having an adequately sized GP workforce seems unlikely to be realised in the near future ${ }^{4,5}$ consequently, new models of care need to be considered.

The pharmacist's role has evolved beyond dispensing medications, with community pharmacists providing various services such as smoking cessation and weight management. ${ }^{6}$ However, as a growing workforce ${ }^{7}$ with a range of skills applicable to primary care, pharmacists remain underutilised. Working in primary care teams, pharmacists can:

- improve prescribing safety;

- support clinical staff in medication audit;

- manage repeat prescriptions; and

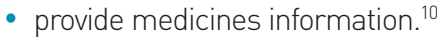

They also have a role in other patientfacing aspects of primary care, including chronic disease management and the treatment of minor illnesses. ${ }^{11,12}$

Previous systematic reviews have considered clinical and patient outcomes, such as blood pressure control and patient satisfaction, ${ }^{11,13}$ but the impact of pharmacists based in primary care on

B Hayhoe, LLM, MD, MRCGP, DRCOG, DPMSA, clinical lecturer in primary care; K Foley, BEd, MSc, PhD, research associate; A Majeed, MD. FRCP, FRCGP, FFPH, professor of primary care; J Ruzangi, BA, MSc, research assistant; G Greenfield, MPH, PhD, research fellow in public health, Department of Primary Care and Public Health; J Acuyo Cespedes, MPH, graduate of the MPH programme, Imperial College London, London.

\section{Address for correspondence}

Benedict Hayhoe, Department of Primary Care and Public Health, School of Public Health, Faculty of health systems remains unclear. One review examined pharmacists' impact on health systems, but did not focus specifically on primary care; ${ }^{14}$ an older review included community pharmacists rather than only those in primary care practices. ${ }^{15}$

The authors sought to understand the impact of integrating pharmacists into primary care teams on health systems indicators, specifically those of utilisation and costs.

\section{METHOD}

A systematic review was conducted in line with recommendations in the Cochrane Handbook for Systematic Reviews of Interventions ${ }^{16}$ and reported according to the Preferred Reporting Items for Systematic Reviews and Meta-Analysis (PRISMA) statement. $^{17}$

\section{Search strategy}

The electronic databases Embase, MEDLINE, Scopus, CINAHL, Health Management Information Consortium (HMIC), and Cochrane Central Register of Controlled Trials (CENTRAL) were searched for articles published between 1947 and June 2018. Medical Subject Headings (MeSH) were used where appropriate. A previous review ${ }^{11}$ helped to guide the searches. Search terms are available from the authors on request.
Medicine, Imperial College London, The Reynolds Building, St Dunstan's Road, London W6 8RP, UK. Email: b.hayhoedimperial.ac.uk

Submitted: 16 January 2019; Editor's response: 2 April 2019; final acceptance: 18 April 2019.

\section{CBritish Journal of General Practice}

This is the full-length article (published online 28 Aug 2019) of an abridged version published in print. Cite this version as: Br J Gen Pract 2019; DOI: https://doi.org/10.3399/bjgp19X705461 


\section{How this fits in}

Evidence shows that pharmacists working in primary care teams can improve clinical outcomes and increase patients' level of satisfaction with their care. However, pharmacists' impact on healthcare systemrelated outcomes is unclear. This review indicates that pharmacists integrated into primary care may reduce the number of GP appointments needed, as well as emergency department attendance; however, they appear to increase primary care use overall. Further research is needed to establish the long-term impact of the integration of pharmacists at scale.

\section{Eligibility criteria}

Randomised controlled trials (RCTs) and observational studies were included, in which pharmacists regularly delivered nondispensing services to individual patients face to face, by telephone, or by screening their medications while in a primary care practice. Studies in which community pharmacists provided a service remotely were excluded. Studies were required to compare the presence and absence of a pharmacist, either by a control group or a baseline comparison.

Only studies examining health system outcomes were included. These are reflected in the NHS pharmacists in primary care pilot, ${ }^{18}$ which considers healthcare utilisation and costs. Studies published in languages other than English were excluded.

\section{Study selection}

Following deduplication, titles and abstracts were screened independently by two authors, before full-text screening against the inclusion criteria was conducted. Disagreements were resolved by consensus with two further authors.

\section{Data items, collection, and extraction}

Data were extracted on outcomes relating to:

- healthcare use: GP visits, medications, hospitalisations, and ED use; and

- healthcare cost: overall healthcare expenditure, medication costs, and hospitalisation and ED visit costs.

\section{Risk of bias assessment}

For RCTs, the Cochrane risk of bias quality assessment tool was used $;^{19}$ the National Institute of Health National Heart, Lung, and Blood Institute quality assessment tool for observational cohort and crosssectional studies was used for observational studies. ${ }^{20}$ Assessment was carried out by the two authors who screened the title and abstracts, and 10 papers were assessed for standardisation by the two authors who resolved the issues of consensus.

\section{Data extraction and synthesis}

Due to the heterogeneous nature of the included studies, meta-analysis was not possible. Descriptive narrative synthesis was used to draw conclusions from extracted data on the effects on the health system of integrating pharmacists into primary care.

\section{RESULTS}

\section{Characteristics of included studies}

Searches identified 3058 studies; of these, 28 were included for data synthesis (Figure 1). Table 1 summarises the characteristics of included studies. These were conducted between 1987 and 2018, and recruited more than 32000 patients. Fourteen studies were from the US, ${ }^{21-34}$ four from Canada, ${ }^{35-38}$ five from the $U K,{ }^{39-43}$ one from Sweden, ${ }^{44}$ one from Spain, ${ }^{45}$ two from Brazil, 46,47 and one from Singapore. ${ }^{48}$ Two studies ${ }^{40,41}$ were further analyses performed on data from previous studies; the original studies 49,50 had no distinct findings on the relevant outcomes, so only the subsequent analyses were included in the final 28 studies. Another two included studies 37,38 were analyses of the same study, but considered 


\section{Table 1. Characteristics and key findings of included studies}

\begin{tabular}{|c|c|c|c|c|c|c|c|}
\hline Author(s) & Year & Country & Study design & Sample size & Population/sample demographic & Health system outcome(s) & Key findings/conclusions \\
\hline $\begin{array}{l}\text { Borenstein } \\
\text { et } a l^{32}\end{array}$ & 2003 & US & $\begin{array}{c}\text { Randomised } \\
\text { comparative trial }\end{array}$ & 197 & $\begin{array}{l}\text { Patients aged }>18 \text { years with } \\
\text { uncontrolled hypertension with } \\
\text { capitated health insurance } \\
\text { recruited from two primary } \\
\text { care offices }\end{array}$ & $\begin{array}{l}\text { Primary care visits, } \\
\text { primary care costs, } \\
\text { medication costs }\end{array}$ & $\begin{array}{l}\text { Physician-pharmacist } \\
\text { comanagement for patients with } \\
\text { uncontrolled hypertension } \\
\text { resulted in improved BP control } \\
\text { and reduced primary care physician } \\
\text { visit rates, and reduced average visit } \\
\text { costs per patient. }\end{array}$ \\
\hline $\begin{array}{l}\text { Britton and } \\
\text { Lurvey }^{28}\end{array}$ & 1991 & US & RCT & 572 & $\begin{array}{l}\text { Patients registered at a primary } \\
\text { care medical centre who were } \\
\text { receiving } \geq 5 \text { prescription or } \\
\text { non-prescription medications }\end{array}$ & $\begin{array}{l}\text { Number of medications, } \\
\text { medication costs, medical } \\
\text { supplies }\end{array}$ & $\begin{array}{l}\text { Medication profile review by a } \\
\text { clinical pharmacist statistically } \\
\text { significantly reduced both the number } \\
\text { and the cost of drugs for patients } \\
\text { receiving } \geq 5 \text { medications }\end{array}$ \\
\hline $\begin{array}{l}\text { Brunisholz } \\
\text { et al }^{33}\end{array}$ & 2018 & US & $\begin{array}{l}\text { Retrospective } \\
\text { observational } \\
\text { study }\end{array}$ & 1358 & $\begin{array}{l}\text { Patients with high BP and/or } \\
\text { diabetes mellitus within a } \\
\text { primary healthcare network }\end{array}$ & $\begin{array}{l}\text { Hospitalisations, ED visits. } \\
\text { primary care visits, } \\
\text { specialty visits }\end{array}$ & $\begin{array}{l}\text { Pharmacist intervention was } \\
\text { associated with improved disease } \\
\text { management, but statistically } \\
\text { significantly increased visits to } \\
\text { primary care, specialty care, care } \\
\text { managers (registered nurse), } \\
\text { and the ED }\end{array}$ \\
\hline Bush et al ${ }^{43}$ & 2018 & UK & $\begin{array}{l}\text { Retrospective } \\
\text { observational } \\
\text { study }\end{array}$ & $\begin{array}{c}5.4 \text { WTE } \\
\text { pharmacists }\end{array}$ & $\begin{array}{l}\text { Clinical pharmacists within } \\
49 \text { GP practices in Dudley CCG }\end{array}$ & $\begin{array}{l}\text { Primary care visits, } \\
\text { healthcare costs }\end{array}$ & $\begin{array}{l}\text { In a 4-month period, pharmacists } \\
\text { saved } 628 \text { GP appointments, } 647 \mathrm{GP} \\
\text { hours in medication review/repeat } \\
\text { prescribing and led to a total savings } \\
\text { of approximately } € 1.5 \text { million }\end{array}$ \\
\hline 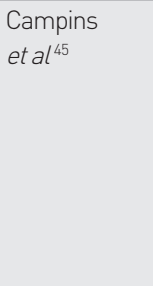 & 2017 & Spain & RCT & 503 & $\begin{array}{l}\text { Community-dwelling } \\
\text { polymedicated ( }>8 \text { drugs) } \\
\text { older people (aged }>70 \text { years) }\end{array}$ & $\begin{array}{l}\text { Number of medications, } \\
\text { primary care visits, ED } \\
\text { visits, hospitalisations, } \\
\text { medication costs, } \\
\text { healthcare costs }\end{array}$ & $\begin{array}{l}\text { Intervention reduced medication use } \\
\text { by } 5 \% \text { with no observed health risks. } \\
\text { There were more primary care visits } \\
\text { with the intervention (difference } \\
\text { became non-significant at } 12 \text { months), } \\
\text { but no differences in ED visits or } \\
\text { hospitalisations. The intervention led } \\
\text { to a } 7 \% \text { reduction in medication costs }\end{array}$ \\
\hline Finley et $a{ }^{27}$ & 2003 & US & RCT & 125 & $\begin{array}{l}\text { Patients registered at a primary } \\
\text { care medical centre, recently } \\
\text { started on antidepressants } \\
\text { for depressive symptoms }\end{array}$ & $\begin{array}{l}\text { Primary care visits, ED } \\
\text { visits, healthcare use, } \\
\text { medication costs }\end{array}$ & $\begin{array}{l}\text { Clinical pharmacists had a favourable } \\
\text { effect on multiple aspects of patient } \\
\text { care, but did not show a statistically } \\
\text { significant difference in resource use }\end{array}$ \\
\hline Harris et al ${ }^{30}$ & 2009 & US & $\begin{array}{l}\text { Prospective } \\
\text { observational } \\
\text { cohort study }\end{array}$ & 92 & $\begin{array}{l}\text { Patients registered at a university } \\
\text { based family medicine clinic, had } \\
\geq 5 \text { medications, multiple medical } \\
\text { conditions, and/or medical } \\
\text { conditions that resulted in high } \\
\text { use of health care }\end{array}$ & Number of medications & $\begin{array}{l}\text { Medication therapy review and } \\
\text { intervention by pharmacist resulted } \\
\text { in a small reduction in average } \\
\text { number of medications per patient }\end{array}$ \\
\hline Hirsch et al ${ }^{21}$ & 2014 & US & $\begin{array}{l}\text { Randomised } \\
\text { pragmatic trial }\end{array}$ & 166 & $\begin{array}{l}\text { Patients with uncontrolled } \\
\text { hypertension registered at a } \\
\text { university-based general } \\
\text { medicine clinic }\end{array}$ & Primary care visits & $\begin{array}{l}\text { Pharmacist intervention was more } \\
\text { effective at lowering BP than usual } \\
\text { care, and associated with fewer } \\
\text { primary care physician visits }\end{array}$ \\
\hline Hunt et $a l^{22}$ & 2008 & US & RCT & 463 & $\begin{array}{l}\text { Patients with uncontrolled } \\
\text { hypertension registered at } \\
\text { one of nine primary care clinics } \\
\text { within a primary care } \\
\text { research network }\end{array}$ & $\begin{array}{l}\text { Primary care visits, } \\
\text { number of medications, } \\
\text { use of generic medications }\end{array}$ & $\begin{array}{l}\text { Patients with pharmacist hypertension } \\
\text { management had more total primary } \\
\text { care visits, but fewer primary care } \\
\text { physician visits. Intervention patients } \\
\text { were statistically significantly more } \\
\text { likely to be prescribed generic } \\
\text { antihypertensive medications but } \\
\text { there was no statistically significant } \\
\text { effect on overall pill burden }\end{array}$ \\
\hline $\begin{array}{l}\text { Lenander } \\
\text { et al }{ }^{44}\end{array}$ & 2014 & Sweden & RCT & 209 & $\begin{array}{l}\text { Patients registered at a large } \\
\text { primary care centre, aged } \\
\geq 65 \text { years with } \geq 5 \text { different } \\
\text { medications }\end{array}$ & $\begin{array}{l}\text { Hospitalisations, primary } \\
\text { care visits, number } \\
\text { of medications }\end{array}$ & $\begin{array}{l}\text { Pharmacist intervention resulted } \\
\text { in reduction in the number of } \\
\text { drugs per patient }\end{array}$ \\
\hline
\end{tabular}




\section{Table 1 continued. Characteristics and key findings of included studies}

\begin{tabular}{|c|c|c|c|c|c|}
\hline Author(s) & Year & Country & y Study design $S$ & Sample size & Population/sample demographic \\
\hline Lowrie et $a l^{39}$ & 2012 & UK C & $\begin{array}{l}\text { Cluster-randomised } \\
\text { controlled, event } \\
\text { driven, trial }\end{array}$ & 2164 & $\begin{array}{l}\text { Patients registered at } 174 \text { NHS } \\
\text { general practices. Eligible patients } \\
\text { were aged } \geq 18 \text { years and had } \\
\text { left ventricular systolic dysfunction } \\
\text { confirmed by cardiac imaging }\end{array}$ \\
\hline Mourão et al ${ }^{47}$ & 2013 & Brazil & RCT & 100 & $\begin{array}{l}\text { Patients registered at six public } \\
\text { health system primary care } \\
\text { centres, aged } \geq 18 \text { years, using } \\
\text { oral antidiabetic medications, and } \\
\text { had presenting HbA1c levels } \\
\text { of } \geq 7 \%\end{array}$ \\
\hline Neilson et al ${ }^{40}$ & 2015 & UK & Pilot RCT & 125 & $\begin{array}{l}\text { Patients registered at six general } \\
\text { practices, aged } \geq 18 \text { years, and } \\
\text { receiving regular prescribed } \\
\text { medication for pain }\end{array}$ \\
\hline $\begin{array}{l}\text { Obreli-Neto } \\
\text { et al }{ }^{46}\end{array}$ & 2015 & Brazil & RCT & 200 & $\begin{array}{l}\text { Patients registered at a primary } \\
\text { healthcare unit, aged } \geq 60 \text { years, } \\
\text { diagnosed with diabetes or } \\
\text { hypertension, and receiving } \\
\text { drug treatment for diabetes or } \\
\text { hypertension }\end{array}$ \\
\hline
\end{tabular}

Health system outcome(s) Key findings/conclusions

A pharmacist intervention resulted in

modest improvements in the prescribing of disease-modifying medications. There was no difference in hospitalisation between groups

The number of drugs taken by the control group remained the same, while the intervention group showed a statistically significant increase

Primary care visits, specialist visits,

Pharmacist-led intervention for chronic pain was more costly and hospitalisations, medication provided similar QALYs to treatment costs, specialist visit costs, as usual hospitalisation costs, primary care costs Primary care visits, specialist visits, ED visits, number of medications, healthcare cost

\section{Pharmacist intervention was} associated with statistically significantly higher GP and specialist visit rates, but statistically significantly lower ED visit rates. There was no difference in overall healthcare costs for intervention and control groups.

\begin{tabular}{|c|c|c|c|c|c|c|}
\hline $\begin{array}{l}\text { Okamoto and } \\
\text { Nakahiro }^{31}\end{array}$ & 2001 & US & $\begin{array}{l}\text { Prospective, } \\
\text { randomised, } \\
\text { comparative study }\end{array}$ & 330 & $\begin{array}{l}\text { Patients in hypertension and } \\
\text { general medicine clinics within } \\
\text { a managed care organisation, } \\
\text { who were } \geq 18 \text { years old and } \\
\text { diagnosed with hypertension, } \\
\text { and taking specified } \\
\text { antihypertensive drugs }\end{array}$ & $\begin{array}{l}\text { Number of medications, } \\
\text { primary care visits, } \\
\text { ED visits, hospitalisations, } \\
\text { medication costs, specialist } \\
\text { visit costs, ED costs, } \\
\text { hospitalisation costs }\end{array}$ \\
\hline
\end{tabular}

There were more primary care visits in the pharmacist managed group, with associated higher visit costs. There were four ED visits in the control group, and none in the pharmacist group. BP measurements were statistically significantly lower in the pharmacist-managed group with cost-effectiveness ratios for $\mathrm{BP}$ mangement lower in the pharmacist intervention group.

\begin{tabular}{|c|c|c|c|c|c|c|c|}
\hline Phelan et a $l^{41}$ & 2008 & UK & RCT & 106 & $\begin{array}{l}\text { Patients aged } \geq 55 \text { years } \\
\text { registered with one of } 15 \\
\text { participating general practices, } \\
\text { who consulted their GP with } \\
\text { pain, stiffness, or both in one } \\
\text { or both knees }\end{array}$ & Medication costs & $\begin{array}{l}\text { Pharmacists can make a positive } \\
\text { contribution to the management of } \\
\text { patients with knee pain in primary } \\
\text { care; pharmacist intervention may } \\
\text { reduce medication-related costs }\end{array}$ \\
\hline $\begin{array}{l}\text { Price-Haywood } \\
\text { et } \text { l }^{34}\end{array}$ & 2017 & US & $\begin{array}{c}\text { Retrospective } \\
\text { observational study }\end{array}$ & 5044 & $\begin{array}{l}\text { Adult patients (>18 years) } \\
\text { with diabetes and/or } \\
\text { hypertension who attended } \\
\text { a community health centre }\end{array}$ & Primary care visits & $\begin{array}{l}\text { Patients who saw a pharmacist } \\
\text { within the collaborative care model } \\
\text { saw their primary care provider more } \\
\text { often. The intervention did not lead to } \\
\text { any differences in BP or } \\
\text { glucose control }\end{array}$ \\
\hline $\begin{array}{l}\text { Ragucci } \\
\text { et } a l^{26}\end{array}$ & 2005 & US & $\begin{array}{l}\text { 1-year observational } \\
\text { study }\end{array}$ & 191 & $\begin{array}{l}\text { Patients with diabetes at three } \\
\text { university-based primary } \\
\text { care clinics }\end{array}$ & Healthcare costs & $\begin{array}{l}\text { Pharmacists achieved significant } \\
\text { improvements in } \mathrm{HbA} 1 \mathrm{c} \text { values, } \mathrm{BP} \text {, } \\
\text { and aspirin use. Statistically } \\
\text { significant cost avoidance was } \\
\text { calculated based on } \mathrm{HbA} 1 \mathrm{c} \text { reductions }\end{array}$ \\
\hline Roth et al ${ }^{29}$ & 2013 & US & $\begin{array}{l}\text { Prospective, } \\
\text { observational } \\
\text { pilot study }\end{array}$ & 64 & $\begin{array}{l}\text { Patients registered at a } \\
\text { community-based primary care } \\
\text { medical practice, aged } \geq 65 \text { years, } \\
\text { who were taking at } \geq 5 \\
\text { medications }\end{array}$ & Hospitalisations, ED visits & $\begin{array}{l}\text { A pharmacist intervention resulted in } \\
\text { a statistically significant } \\
\text { reduction in medication-related } \\
\text { problems, and a non-significant } \\
\text { reduction in acute health services use }\end{array}$ \\
\hline
\end{tabular}




\section{Table 1 continued. Characteristics and key findings of included studies}

\begin{tabular}{|c|c|c|c|c|c|c|c|}
\hline Author(s) & Year & Country & Study design & Sample size & Population/sample demographic & Health system outcome(s) & Key findings/conclusions \\
\hline $\begin{array}{l}\text { Rothman } \\
\text { et al }{ }^{23}\end{array}$ & 2005 & US & RCT & 217 & $\begin{array}{l}\text { Patients registered at a university } \\
\text { general internal medicine } \\
\text { practice with type } 2 \text { diabetes } \\
\text { and poor glycaemic control } \\
\text { (HbAlc level } \geq 8.0 \% \text { ) }\end{array}$ & $\begin{array}{l}\text { Primary care visits, urgent } \\
\text { care visits, hospitalisations }\end{array}$ & $\begin{array}{l}\text { Pharmacist intervention resulted in } \\
\text { statistically significant improvement } \\
\text { in BP and glycaemic control, but had } \\
\text { no statistically significant } \\
\text { impact on the use of clinical services }\end{array}$ \\
\hline $\begin{array}{l}\text { Rudd and } \\
\text { Dierr }^{24}\end{array}$ & 2010 & US & $\begin{array}{l}\text { Retrospective } \\
\text { medical record } \\
\text { review }\end{array}$ & 996 & $\begin{array}{l}\text { Patients registered at } 25 \\
\text { outreach primary care health } \\
\text { centres, receiving long-term } \\
\text { anticoagulation therapy with } \\
\text { warfarin }\end{array}$ & Hospitalisations, ED visits & $\begin{array}{l}\text { Pharmacist anticoagulation } \\
\text { management services resulted in a } \\
\text { statistically significant reduction in } \\
\text { rates of anticoagulation-related ED } \\
\text { visits and hospitalisations, with } \\
\text { financial impact through avoided costs. }\end{array}$ \\
\hline $\begin{array}{l}\text { Sellors } \\
\text { et al }{ }^{35}\end{array}$ & 2001 & $\begin{array}{r}\text { Canada } \\
0\end{array}$ & $\begin{array}{l}\text { Randomised, } \\
\text { observer-blinded, } \\
\text { controlled trial }\end{array}$ & 132 & $\begin{array}{l}\text { Patients aged } \geq 65 \text { years from } \\
\text { four family practices, who were } \\
\text { taking } \geq 4 \text { regular medications }\end{array}$ & $\begin{array}{l}\text { Number of medications, } \\
\text { medication costs }\end{array}$ & $\begin{array}{l}\text { Results suggested a statistically } \\
\text { non-significant trend towards } \\
\text { lower medication costs through face- } \\
\text { to-face medication reviews carried out } \\
\text { by pharmacists }\end{array}$ \\
\hline $\begin{array}{l}\text { Sellors } \\
\text { et al }{ }^{36}\end{array}$ & 2003 & Canada & RCT & $\begin{array}{l}48 \text { family } \\
\text { physicians } \\
\text { and } 889 \\
\text { patients }\end{array}$ & $\begin{array}{l}\text { Patients } \geq 65 \text { years, taking } \\
\geq 5 \text { medications, registered in } \\
24 \text { family practices }\end{array}$ & $\begin{array}{l}\text { Number of medications, } \\
\text { medication costs, } \\
\text { healthcare costs }\end{array}$ & $\begin{array}{l}\text { The intervention did not demonstrate } \\
\text { any statistically significant effect on } \\
\text { health system outcomes. Physicians } \\
\text { were receptive to recommendations } \\
\text { on drug-related problems, suggesting } \\
\text { feasibility of collaboration between } \\
\text { physicians and pharmacists }\end{array}$ \\
\hline Siaw et al ${ }^{48}$ & 2017 & Singapore & RCT & 411 & $\begin{array}{l}\text { High-risk patients aged } \\
\geq 21 \text { years with uncontrolled } \\
\text { type } 2 \text { diabetes, polypharmacy, } \\
\text { and comorbidities; registered } \\
\text { at four outpatient healthcare } \\
\text { institutions }\end{array}$ & $\begin{array}{l}\text { Primary care visits, visit } \\
\text { costs, laboratory test costs, } \\
\text { medication costs }\end{array}$ & $\begin{array}{l}\text { Pharmacist intervention was } \\
\text { associated with fewer physician } \\
\text { visits, but slightly higher visit costs } \\
\text { due to additional pharmacists visits. } \\
\text { Overall healthcare costs were lower } \\
\text { with pharmacist intervention, with } \\
\text { medication costs the greatest } \\
\text { contributor to this reduction. }\end{array}$ \\
\hline $\begin{array}{l}\text { Simpson } \\
\text { et } \text { el }^{38}\end{array}$ & 2015 & Canada & RCT & 123 & $\begin{array}{l}\text { Patients with type } 2 \text { diabetes } \\
\text { registered at five primary care } \\
\text { clinics }\end{array}$ & $\begin{array}{l}\text { ED visits, hospitalisations, } \\
\text { specialist visits, healthcare } \\
\text { contacts }\end{array}$ & $\begin{array}{l}\text { Adding pharmacists to primary care } \\
\text { teams was a cost-effective strategy for } \\
\text { reducing cardiovascular risk in } \\
\text { patients with type } 2 \text { diabetes }\end{array}$ \\
\hline $\begin{array}{l}\text { Stergachis } \\
\text { et al }{ }^{25}\end{array}$ & 1987 & US & RCT & 17 physicians & $\begin{array}{l}\text { Two clinical pharmacists } \\
\text { working with patients registered } \\
\text { in two family practices }\end{array}$ & Medication costs & $\begin{array}{l}\text { Pharmacists were unable to produce } \\
\text { statistically significant change in } \\
\text { medication costs. This programme } \\
\text { was not economically self-sustaining } \\
\text { during the first } 6 \text { months }\end{array}$ \\
\hline $\begin{array}{l}\text { Zermansky } \\
\text { et al }{ }^{42}\end{array}$ & 2001 & UK & RCT & 1188 & $\begin{array}{l}\text { Patients registered at four } \\
\text { general practices, aged } \\
\geq 65 \text { years, receiving at least } \\
\text { one repeat prescription }\end{array}$ & $\begin{array}{l}\text { Number of medications, } \\
\text { medication costs, primary } \\
\text { care visits, specialist visits, } \\
\text { hospitalisations }\end{array}$ & $\begin{array}{l}\text { Monthly medication costs rose less in } \\
\text { the pharmacist intervention group. } \\
\text { Patients in the intervention group also } \\
\text { had a statistically smaller rise in the } \\
\text { number of medications prescribed } \\
\text { than in the control group. }\end{array}$ \\
\hline
\end{tabular}

$B P=$ blood pressure. $C C G=$ clinical commissioning group. $E D=$ emergency department. NHS = National Health Service. QALY =quality-adjusted life year. RCT = randomised controlled trial. WTE = whole-time equivalent.

separate relevant outcomes. Seven studies were observational, $24,26,29,30,33,34,43$ and 21 were RCTs. ${ }^{21-23,25,27,28,31,32,35-42,44-48}$
All of the included studies examined pharmacists working in primary care; however, the type of service offered varied, 
and can be broadly divided into three categories:

- medication review: the pharmacist reviewed patients' medications and offered suggestions to clinicians; ${ }^{21-48}$

- treatment management: the pharmacist managed patients' treatment, including ordering laboratory tests, seeing patients regularly, and, in some cases, prescribing medication; $21,22,24,26,27,31-34,37,38,40,41,43,46,47$ and;

- patient education: the pharmacist educated patients on lifestyle factors or behaviours to improve their health. ${ }^{21-23,25-27,29,32-34,44,46,47}$

Pharmacists in some studies offered more than one of these services. No study compared different pharmacist interventions, so relative impact could not be assessed.

\section{Risk of bias assessment}

Five of the seven observational studies were of fair quality; ${ }^{24,26,29,30,33}$ the remaining two were of poor quality; 34,43 none provided sample-size justification or blinded outcome assessors. Experimental studies were all of fair overall quality, but all introduced a high risk of bias in failing to blind participants. ${ }^{21-23,25,27,28,31,32,35-42,44-48}$ In addition, in all but two studies ${ }^{36,39}$ there was contamination between groups, with GPs seeing patients in both the control and intervention groups. Most RCTs did not state whether outcome assessors were blinded to group allocation.

\section{Impact of pharmacists in primary care}

The impact of pharmacist integration into primary care on healthcare utilisation and cost is summarised in Table 1. In common with much of the literature, the term 'visit' is used to refer to occasions when patients see health professionals in primary care clinics or hospital departments. Domiciliary visiting was not considered in this review.

\section{Primary care visits}

Nine studies assessed the impact of the integration of pharmacists on GP visit rates; $21,22,32,34,40,42,43,46,48$ four showed a statistically significant decrease of approximately two GP visits per patient per year with the integration of pharmacists. ${ }^{21,22,32,48}$ One UK study ${ }^{43}$ reported 628 GP appointments and 647 hours of GP administrative time saved over a 4-month period through the integration of 5.4 whole-time equivalent (WTE) pharmacists across $49 \mathrm{GP}$ practices. Two further studies showed small increases in
GP visit rates, ${ }^{34,46}$ and two demonstrated no statistically significant difference. ${ }^{40,42}$ Of 10 studies considering overall primary care visits, 21-23,27,31-33,40,44,45 including appointments with pharmacists and other primary care professionals, four found an increase in primary care usage following the integration of pharmacists, 22,31,33,45 and one found that pharmacists scheduled nearly four times as many appointments as GPs. ${ }^{31}$ The remaining six studies found no statistically difference in overall primary care use. ${ }^{21,23,27,32,40,44}$

\section{Secondary care visits}

Six studies assessed the consulting of secondary care professionals (specialist and outpatient department visits).33,37,38,40,42,46 In one of these, pharmacist intervention was associated with statistically significantly more ambulatory clinical visits, including secondary care visits; ${ }^{33}$ another showed a small statistically significant increase in specialist appointments (0.2 versus 0.1 mean visits per patient in intervention and control groups respectivelyl associated with pharmacist integration. ${ }^{46}$ No other significant differences were identified.

\section{Overall healthcare contacts}

Three studies assessed overall healthcare contacts, ${ }^{27,37,38}$ showing increasing trends in healthcare use with pharmacist intervention. However, this was significant in only one study, ${ }^{37}$ in which $78 \%$ of contacts were protocol driven or interim follow-up appointments with pharmacists (twothirds of these interim contacts were by telephone).

\section{Medication use}

Eleven studies assessed the number of medications per patient. 22,28,30,31,35,36,42,44-47 Findings were mixed. Two studies showed small statistically significant increases in medications prescribed in pharmacist interventions, ${ }^{42,47}$ lin one of these, medications increased in both intervention and control groups, but to a lesser extent with the intervention ${ }^{42}$ ). Four studies showed small statistically significant decreases in medication use with pharmacist intervention, $28,30,44,45$ while the remainder showed no statistically significant effect on overall numbers of medications. ${ }^{22,31,35,36,46}$

\section{Hospitalisation and ED use}

Twelve studies assessed the number of hospitalisations or length of stay in hospital, 23,24,29,31,33,37-40,42,44,45 and 10 examined ED visits (including urgent care). ${ }^{23,24,27,29,31,33}$. $37,38,45,46$ Only one study, in which pharmacists managed anticoagulation 
in primary care, showed a statistically significant reduction in hospitalisations with pharmacist intervention. ${ }^{24}$ No studies reported a statistically significant impact on length of hospital stay. Three studies $24,31,46$ showed a significant reduction in ED use with pharmacist intervention, alongside a number with non-significant trends in this direction; $23,27,29,38$ one study showed a significant increase in ED use. ${ }^{33}$

\section{Medication costs}

Twelve studies examined medication costs, ${ }^{25,27,28,31,32,35,36,40-42,45,48}$ with three showing statistically significant decreases in spending on medication, ${ }^{28,42,45}$ one suggesting a statistically non significant trend in this direction, ${ }^{35}$ and one a statistically smaller increase in spending with pharmacist intervention than with controls. ${ }^{42}$ One further study ${ }^{22}$ considered prescribing generic versions of medications as a surrogate for a reduction in medicationrelated costs, showing significantly higher rates of generic prescribing in the pharmacist intervention group.

\section{Primary and secondary care utilisation costs}

Ten studies assessed the cost of healthcare utilisation, 26,31,32,36,37,40,43,45,46,48 looking at outcomes including secondary care clinic costs, laboratory tests, and primary care costs. Three identified cost increases associated with pharmacist intervention that related to increased clinic appointments in both primary and secondary care..$^{31,46,48}$ However, significant healthcare cost decreases were identified in some studies, including lower average cost per visit, ${ }^{32,48}$ less spending on laboratory tests, ${ }^{48}$ lower total cost for diabetes care, ${ }^{48}$ and reduced GP hours spent on medication review and repeat prescribing: ${ }^{43}$ this last study estimated annual cost savings of $€ 1.5$ million through the integration of 5.4 WTE pharmacists across 49 GP practices.

\section{Hospitalisation and ED visit costs}

Three studies considered hospitalisation costs. $.4,31,40$ No related outcomes of statistical significance were reported, but Rudd and Dier's study ${ }^{24}$ estimated a large saving in hospitalisation avoidance lbased on reduced admission rates compared with usual physician care). Two studies assessed ED costs; ${ }^{24,31}$ both reported statistically significantly fewer patients attending the ED in the intervention groups and large cost savings were estimated based on these visit frequencies.

\section{DISCUSSION}

Summary

Integrating pharmacists into primary care was likely to reduce the number of GP appointments; however, it may have increased the overall use of primary care through relatively frequently scheduled appointments with pharmacists. Pharmacists in primary care did not appear to affect hospitalisations, but their integration was likely associated with fewer ED attendances. Evidence relating to the impact on the number of prescribed medications per patient was mixed but, in a few studies, pharmacist interventions were associated with a reduction in medicationrelated spending. There was some evidence of a reduction in overall expenditure on both primary and secondary health care, and several studies estimated cost reductions based on decreases in ED use.

\section{Strengths and limitations}

This review provides a clearer sense of the potential impact pharmacists in primary care can have on a health system. Both RCTs and observational studies were included, increasing the reliability of the evidence considered as recommended by Concato et al..$^{51}$ However, included studies were all of fair or poor quality, with several important sources of bias. No study blinded participants to the status of the professionals they consulted, although this would, of course, have been difficult to achieve in practice. Like most literature reviews, it is possible that the one presented here is affected by a degree of publication bias in terms of the studies that were included. For practical reasons, only studies published in English were included, potentially resulting in exclusion bias.

The small numbers of patients involved in many studies make it difficult to draw firm conclusions about the impact on healthcare services. This is particularly the case for those estimating cost savings through hospitalisation or ED visit avoidance. As an example, one study ${ }^{31}$ reported no ED visits in the intervention group $(n=166)$, compared with four in the physician-managed group ( $n=164)$ over a 6 -month period.

\section{Comparison with existing literature}

Previous reviews have demonstrated the effectiveness of pharmacists based in primary care practices at improving various clinical outcomes, with most evidence relating to cardiovascular disease, diabetes, and avoidance of drug-related errors. ${ }^{11}$ A previous systematic review of pharmacist services in primary care in low- and 
middle-income countries ${ }^{14}$ concluded that pharmacists may reduce healthcare use including GP visit rates and hospitalisation - as well as medication-related costs, but noted the low quality of the evidence. A number of studies of pharmacists in primary care have reported cost-effectiveness via an improvement in clinical outcomes, such as cardiovascular risk, ${ }^{52}$ and a reduction in drug-related errors. ${ }^{8}$

Findings of included studies were conflicting in terms of the impact on the number of medications per patient and overall medication costs: some showed an increase in medication use following pharmacist intervention. A previous systematic review of non-patient-facing pharmacist interventions in primary care suggested that pharmacists could improve the appropriateness of prescribing, ${ }^{53}$ it may be that increases in prescribed medications result from pharmacists starting treatments appropriately, and that the observed reduction in medication-related costs is effected by switching to generic or more cost-effective medications.

The degree of integration of pharmacists into primary care teams may have a marked impact on their effectiveness: a recent systematic review ${ }^{13}$ found that a higher degree of pharmacist integration was associated with improved health outcomes. It is possible that greater integration also has an impact on health system outcomes, along with the type of pharmacist intervention (medication review, treatment management, or patient education), and the pharmacist's skill-set lincluding their ability to prescribe independently); however, data were not available to allow for assessment of this.

\section{Implications for research and practice}

The potential for pharmacists to reduce the number of GP appointments has important implications at a time of unprecedented demand on the GP workforce in many countries. $^{2}$ Limitations regarding the quality of the included studies and the heterogeneous nature of the pharmacist interventions that were reported make it difficult to draw firm conclusions about the impact on GP workload; however substituting consultations with pharmacists for more-costly GP appointments is likely to have a cost advantage.

The increased overall primary healthcare use found in some studies included in this review may suggest an increased ability of patients to access primary care through pharmacist integration. However, with much of this increase relating to protocol-driven follow-up, the impact on the pressure for primary care appointments outside of study settings is unclear. Furthermore, no study reported actual or perceived GP workload pressure. It is possible that the training, support, and supervision of pharmacists in primary care might represent an additional burden for GPs.

A recent focus on initiatives to reduce pressure on EDs has shown a clear association between accessible primary care services and a reduction in ED attendance ${ }^{54} \mathrm{It}$ is possible that the apparent impact of pharmacists in primary care on ED use relates to improved access to primary care; irrespective of mechanism, any shift in usage from costly emergency services is of significant benefit to wider healthcare systems.

The integration of pharmacists into primary care must overcome a variety of barriers, including professional and patient resistance, ${ }^{55-57}$ as well as more practical issues, such as accommodating additional professionals in crowded facilities; ${ }^{56}$ a recent realist review ${ }^{57}$ highlighted the need for flexibility in terms of approach to suit individual practice needs. However, the successful piloting of a programme to integrate pharmacists into primary care practices in England has resulted in expansion, with ongoing funding for pharmacists in this setting nationwide. ${ }^{58}$ This provides an opportunity for further research, as wider implementation is likely to be necessary to give clear evidence of the impact of pharmacists' integration into primary care on health utilisation and costs. In the context of current workload challenges in primary care, as well as ongoing pressures on unscheduled care, it is particularly important that the impact of pharmacist integration on GP workload and ED use is clarified to inform future policy.

In conclusion, limited evidence suggests that pharmacists in primary care may save GPs time through a reduction in scheduled GP appointments and time spent on medication-related administration, while improving patient access to primary care. The possibility that pharmacists may also reduce ED use and overall healthcare costs suggests that initiatives for pharmacists' integration into primary care are likely to be cost effective. Further research is needed to establish the true impact of the integration of pharmacists in primary care at scale on healthcare systems in the longer term. 


\section{REFERENCES}

1. Hobbs FDR, Bankhead C, Mukhtar T, et al. Clinical workload in UK primary care: a retrospective analysis of 100 million consultations in England, 2007-14 Lancet 2016; 387: 2323-2330.

2. Baird B, Charles A, Honeyman M, et al. Understanding pressures in general practice. 2016. https://www.kingsfund.org.uk/publications/pressures-ingeneral-practice laccessed 1 Aug 2019).

3. NHS England. Five year forward view. 2014. https://www.england.nhs.uk/ wp-content/uploads/2014/10/5yfv-web.pdf laccessed 1 Aug 2019).

4. lacobucci G. GP numbers drop despite government pledge to boost workforce BMJ 2017; 356: j1623.

5. Petterson SM, Liaw WR, Tran C, Bazemore AW. Estimating the residency expansion required to avoid projected primary care physician shortages by 2035. Ann Fam Med 2015; 13(2): 107-114.

6. Brown TJ, Todd A, O'Malley C, et al. Community pharmacy-delivered interventions for public health priorities: a systematic review of interventions for alcohol reduction, smoking cessation and weight management, including meta-analysis for smoking cessation. BMJ Open 2016; 6: e009828.

7. Gall D, Bates I, Bruno A. 2012 FIP global pharmacy workforce report. 2012. http://discovery.ucl.ac.uk/1369202/1/workforce\%20report\%20final.pdf laccessed 1 Aug 2019).

8. Avery AJ, Rodgers S, Cantrill JA, et al. A pharmacist-led information technology intervention for medication errors (PINCER): a multicentre, cluster randomised controlled trial and cost-effectiveness analysis. Lancet 2012; 379(9823): 13101319.

9. Nkansah N, Mostovetsky O, Yu C, et al. Effect of outpatient pharmacists' nondispensing roles on patient outcomes and prescribing patterns. Cochrane Database Syst Rev 2010; 7: CD000336.

10. Komwong D, Greenfield G, Zaman H, Majeed A, Hayhoe B. Clinical pharmacists in primary care: a safe solution to the workforce crisis? J R Soc Med 2018; 111(4): $120-124$

11. Tan EC, Stewart K, Elliott RA, George J. Pharmacist services provided in general practice clinics: a systematic review and meta-analysis. Res Social Adm Pharm 2014; 10(4): 608-622.

12. Santschi V, Chiolero A, Colosimo A, et al. Improving blood pressure control through pharmacist interventions: a meta-analysis of randomized controlled trials. J Am Heart Assoc 2014; 3(2): e000718.

13. Hazen ACM, de Bont AA, Boelman $L$, et al. The degree of integration of nondispensing pharmacists in primary care practice and the impact on health outcomes: a systematic review. Res Social Adm Pharm 2018; 14(3): 228-240.

14. Pande S, Hiller JE, Nkansah N, Bero L. The effect of pharmacist-provided nondispensing services on patient outcomes, health service utilisation and costs in low- and middle-income countries. Cochrane Database Syst Rev 2013; 2: CD010398.

15. Beney J, Bero LA, Bond C. Expanding the roles of outpatient pharmacists: effects on health services utilisation, costs, and patient outcomes. Cochrane Database Syst Rev 2000; 3: CD000336.

16. Higgins JPT, Green S, eds. Cochrane handbook for systematic reviews of interventions. Chichester: Wiley, 2008.

17. Liberati A, Altman DG, Tetzlaff J, et al. The PRISMA statement for reporting systematic reviews and meta-analyses of studies that evaluate healthcare interventions: explanation and elaboration. BMJ 2009; 339: b2700.

18. NHS England. Clinical pharmacists in general practice. https://www.england. nhs.uk/contact-us/privacy-notice/how-we-use-your-information/public-andpartners/clinical-pharmacists-in-general-practice/ laccessed 1 Aug 2019).

19. Higgins JP, Altman DG, Gøtzsche PC, et al. The Cochrane Collaboration's tool for assessing risk of bias in randomised trials. BMJ 2011; 343: $\mathrm{d5928.}$

20. National Institute of Health, National Heart, Lung, and Blood Institute. Quality assessment tool for observational cohort and cross-sectional studies. https:// wnw.nhlbi.nih.gov/health-topics/study-quality-assessment-tools laccessed 1 Aug 2019).

21. Hirsch JD, Steers N, Adler DS, et al. Primary care-based, pharmacist-physician collaborative medication-therapy management of hypertension: a randomized, pragmatic trial. Clin Ther 2014; 36(9): 1244-1254.

22. Hunt JS, Siemienczuk J, Pape G, et al. A randomized controlled trial of teambased care: impact of physician-pharmacist collaboration on uncontrolled hypertension. J Gen Intern Med 2008; 23(12): 1966-1972.
23. Rothman RL, Malone R, Bryant B, et al. A randomized trial of a primary carebased disease management program to improve cardiovascular risk factors and glycated hemoglobin levels in patients with diabetes. Am J Med 2005; 118(3): 276-284.

24. Rudd KM, Dier JG. Comparison of two different models of anticoagulation management services with usual medical care. Pharmacotherapy 2010; 30(4): 330-338.

25. Stergachis A, Fors M, Wagner EH, et al. Effect of clinical pharmacists on drug prescribing in a primary-care clinic. Am J Hosp Pharm 1987; 44(3): 525-529.

26. Ragucci KR, Fermo JD, Wessell AM, Chumney EC. Effectiveness of pharmacist-administered diabetes mellitus education and management services. Pharmacotherapy 2005; 25(12): 1809-1816.

27. Finley PR, Rens HR, Pont JT, et al. Impact of a collaborative care model on depression in a primary care setting: a randomized controlled trial. Pharmacother 2003; 23(9): 1175-1185.

28. Britton M, Lurvey P. Impact of medication profile review on prescribing in a general medicine clinic. Am J Hosp Pharm 1991; 48(2): 265-270.

29. Roth MT, Ivey JL, Esserman DA, et al. Individualized medication assessment and planning: optimizing medication use in older adults in the primary care setting. Pharmacother 2013; 33(8): 787-797.

30. Harris IM, Westberg SM, Frakes MJ, Van Vooren JS. Outcomes of medication therapy review in a family medicine clinic. J Am Pharm Assoc 2009; 49(5): 623-627.

31. Okamoto MP, Nakahiro RK. Pharmacoeconomic evaluation of a pharmacistmanaged hypertension clinic. Pharmacother 2001; 21(11): 1337-1344.

32. Borenstein JE, Graber G, Saltiel E, et al. Physician-pharmacist comanagement of hypertension: a randomized, comparative trial. Pharmacotherapy 2003; 23(2): 209-216.

33. Brunisholz KD, Olson J, Anderson JW, et al. 'Pharming out' support: a promising approach to integrating clinical pharmacists into established primary care medical home practices. J Int Med Res 2018; 46(1): 234-248.

34. Price-Haywood EG, Amering S, Luo Q, Lefante JJ. Clinical pharmacist teambased care in a safety net medical home: facilitators and barriers to chronic care management. Popul Health Manag 2017; 20(2): 123-131.

35. Sellors C, Dalby DM, Howard M, et al. A pharmacist consultation service in community-based family practices: a randomized, controlled trial in seniors. J Pharm Technol 2001; 17(6): 264-269.

36. Sellors J, Kaczorowski J, Sellors C, et al. A randomized controlled trial of a pharmacist consultation program for family physicians and their elderly patients. CMAJ 2003; 169(1): 17-22.

37. Simpson SH, Majumdar SR, Tsuyuki RT, et al. Effect of adding pharmacists to primary care teams on blood pressure control in patients with type 2 diabetes: a randomized controlled trial. Diabetes Care 2011; 34(1): 20-26.

38. Simpson SH, Lier DA, Majumdar SR, et al. Cost-effectiveness analysis of adding pharmacists to primary care teams to reduce cardiovascular risk in patients with type 2 diabetes: results from a randomized controlled trial. Diabet Med 2015; 32(7): 899-906.

39. Lowrie R, Mair FS, Greenlaw N, et al. Pharmacist intervention in primary care to improve outcomes in patients with left ventricular systolic dysfunction. Eur Heart J 2012; 33(3): 314-324.

40. Neilson AR, Bruhn H, Bond CM, et al. Pharmacist-led management of chronic pain in primary care: costs and benefits in a pilot randomised controlled trial. BMJ Open 2015; 5: e006874.

41. Phelan M, Foster NE, Thomas E, et al. Pharmacist-led medication review for knee pain in older adults: content, process and outcomes. Int J Pharm Pract 2008; 16(6): 347-355.

42. Zermansky AG, Petty DR, Raynor DK, et al. Randomised controlled trial of clinical medication review by a pharmacist of elderly patients receiving repeat prescriptions in general practice. BMJ 2001; 323(7325): 1340-1343.

43. Bush J, Langley CA, Jenkins D, et al. Clinical pharmacists in general practice: an initial evaluation of activity in one English primary care organisation. Int J Pharm Pract 2018; 26(6): 501-506.

44. Lenander C, Elfsson B, Danielsson B, et al. Effects of a pharmacist-led structured medication review in primary care on drug-related problems and hospital admission rates: a randomized controlled trial. Scand J Prim Health Care 2014; 32(4): 180-186.

45. Campins L, Serra-Prat M, Gózalo I, et al. Randomized controlled trial of an intervention to improve drug appropriateness in community-dwelling polymedicated elderly people. Fam Pract 2017; 34(1): 36-42. 
46. Obreli-Neto PR, Marusic S, Guidoni CM, et al. Economic evaluation of a pharmaceutical care program for elderly diabetic and hypertensive patients in primary health care: a 36-month randomized controlled clinical trial. J Manag Care Spec Pharm 2015; 21(1): 66-75.

47. Mourão AO, Ferreira WR, Martins MA, et al. Pharmaceutical care program for type 2 diabetes patients in Brazil: a randomised controlled trial. Int J Clin Pharm 2013; 35(1): 79-86.

48. Siaw MYL, Ko Y, Malone DC, et al. Impact of pharmacist-involved collaborative care on the clinical, humanistic and cost outcomes of high-risk patients with type 2 diabetes (IMPACT): a randomized controlled trial. J Clin Pharm Ther 2017: 42(4): 475-482

49. Bruhn H, Bond CM, Elliott AM, et al. Pharmacist-led management of chronic pain in primary care: results from a randomised controlled exploratory trial. BMJ Open 2013; 3(4): e002361.

50. Hay EM, Foster NE, Thomas E, et al. Effectiveness of community physiotherapy and enhanced pharmacy review for knee pain in people aged over 55 presenting to primary care: pragmatic randomised trial. BMJ 2006; 333(7576): 995.

51. Concato J, Shah N, Horwitz RI. Randomized, controlled trials, observational studies, and the hierarchy of research designs. N Engl J Med 2000; 342(25): 1887-1892
52. Polgreen LA, Han J, Carter BL, et al. Cost-effectiveness of a physicianpharmacist collaboration intervention to improve blood pressure control. Hypertension 2015; 66(6): 1145-1151.

53. Riordan DO, Walsh KA, Galvin R, et al. The effect of pharmacist-led interventions in optimising prescribing in older adults in primary care: a systematic review. SAGE Open Med 2016; 4: 2050312116652568

54. Cowling TE, Harris MJ, Watt HC, et al. Access to general practice and visits to accident and emergency departments in England: cross-sectional analysis of a national patient survey. Br J Gen Pract 2014; DOI:https://doi.org/10.3399/ bjgp14X680533.

55. Tan EC, Stewart K, Elliott RA, George J. Stakeholder experiences with general practice pharmacist services: a qualitative study. BMJ Open 2013; 3(9): e003214

56. Tan EC, Stewart K, Elliott RA, George J. Integration of pharmacists into general practice clinics in Australia: the views of general practitioners and pharmacists. Int J Pharm Pract 2014; 22(1): 28-37.

57. Anderson C, Zhan K, Boyd M, Mann C. The role of pharmacists in general practice: a realist review. Res Social Adm Pharm 2019; 15(4): 338-345.

58. NHS England. General practice forward view. 2016. https://www.england.nhs. uk/wp-content/uploads/2016/04/gpfv.pdf laccessed 1 Aug 2019). 